\title{
A Review of Full-endoscopic Interlaminar Discectomy for Lumbar Disc Disease: A Historical and Technical Overview
}

\author{
Yanting Liu', Jin-Sung Kim${ }^{1}$, Chien-Min Chen ${ }^{2}$, Gun $\mathrm{Choi}^{3}$, Sang Ho Lee ${ }^{3}$, Sebastian Ruetten ${ }^{4}$, \\ Xifeng Zhang, ${ }^{5}$ Koichi Sairyo ${ }^{6}$ \\ ${ }^{1}$ Department of Neurosurgery, Seoul St. Mary's Hospital, College of Medicine, The Catholic University of Korea, Seoul, Republic of Korea \\ ${ }^{2}$ Division of Neurosurgery, Department of Surgery, Changhua, Taiwan \\ ${ }^{3}$ Department of Spine Surgery, Wooridul Spine Hospital, Pohang, Republic of Korea \\ ${ }^{4}$ Center for Spine Surgery and Pain Therapy, Center for Orthopaedics and Traumatology of the St. Elisabeth Group-Catholic Hospitals \\ Rhein-Ruhr, St. Anna Hospital Herne/Marien Hospital Herne University Hospital/Marien Hospital Witten, Hospitalstr, Herne, Germany \\ ${ }^{5}$ Department of Orthopedics, The General Hospital of People's Liberation Army, Beijing 100853, China, Beijing, China \\ ${ }^{6}$ Department of Orthopedics, The University of Tokushima
}

Corresponding Author: Jin-Sung Kim, MD, $\mathrm{PhD}$

Department of Neurosurgery, Seoul St. Mary's Hospital, College of Medicine,

The Catholic University of Korea

222 Banpo-daero, Seocho-gu, Seoul

06591, Republic of Korea

TEL: +82-2-2258-6265

FAX: + 82-2-2258-6128

E-mail: mdlukekim@gmail.com; md1david@catholic.ac.kr

Received: February 02, 2021

Revised: February 22, 2021

Accepted: March 05, 2021
Lumbar disc disease is an age or trauma-related disc injury with the clinical symptoms such as lower back pain or sciatica. Surgical treatment is the reliable and effective solution only if the symptoms grow progressively worse and conservative treatments fail. The purpose of spinal surgery is to remove the real pain generator and maintain physiological spinal alignment. Traditionally, patients after discectomy tend to experience the risk of postoperative intervertebral disc degeneration with intractable low back pain. Therefore, the minimally invasive techniques have rapidly developed in the past decades and brought revolutionary progress on techniques modification. Herein, we report the historical overview of the interlaminar fullendoscopic lumbar discectomy and technical evolution based on the review of literature.

Key Words: Interlaminar Endoscopy, Diskectomy, Minimally Invasive Surgical Procedures, Lumbar Vertebrae

\section{INTRODUCTION}

During $70-85 \%$ of people's lifetime, low back pain is a regular torturous feeling which is generally accompanied by sciatica and possible foot numbness or weakness ${ }^{1-3)}$. The most common cause of these symptoms is the intervertebral structure degeneration, leading to the constriction of the spinal canal with a displacement of nucleus, cartilage, fragmented annular tissue exceeding the intervertebral disc area ${ }^{4,5}$. Traditional conservative treatment is the first option for symptomatic lumbar disc herniation patients, such as drugs, physical therapy, or epidural steroid injection ${ }^{2)}$. There have also been reports that oxygen-ozone therapy is used as an interventional spinal procedure to reduce herniated disc symptoms ${ }^{6,7)}$. Once all conservative managements have been exhausted and neurologic symptoms occur as progressive muscle weakness, cauda equina syndromes, or bladder dysfunction, surgical intervention is the only reliable and effective solution ${ }^{8,9)}$.
To address concerns of the trauma and functional recovery after conventional spine surgery, the minimally invasive techniques have rapidly developed in the past decades ${ }^{10,11)}$. Especially the emergence of endoscopy technology has brought revolutionary progress for spinal surgery. This achievement is inseparable from its unique working mechanism and flexible maneuverability. The small-caliber working cannula can reduce the multifidus muscle stripping and further maintain the integrity of motion stabilizer. Working with high-resolution angle lens optics and multichannel irrigation fluid system, the endoscopic system not only provides the visibility of corner areas, but also restrains the intraoperative bleeding and creates an uncluttered surgical field. In addition, the magnified view field enables more elaborate manipulation on the critical neural tissue and further significantly decreases the epidural scarring formation. In recent years, the instrument function has made tremendous progress, such as the sharpness of the camera system, the exceptional power of the dynamical system, and the improved compatibility with navigation systems. 
Transforaminal endoscopic lumbar discectomy, as the earliest operation option to treat disc herniation patients with endoscopy, pass through Kambin's triangle corridor to accomplish the herniated disc removal; however, the transforaminal approach cannot reach every corner around the spinal canal. Then, the emergence of research on the possibility of discectomy via interlaminar approach aroused clinician's concern. Numerous surgeons set about transforming transforaminal full-endoscopic lumbar discectomy (TELD) to interlaminar full-endoscopic lumbar discectomy (IELD) to treat lumbar disc herniation because of its more familiarst posterior anatomical structure than that of the foramen approach. Meanwhile, it also broadens full endoscopic indications on the degenerative lumbar stenosis. In this review article, the authors will report the historical overview of the interlaminar full-endoscopic lumbar discectomy and technical evolution based on the review of literature.

\section{The Historical Overview of IELD}

After the achievement of Lister's antisepsis in open surgery, the first discectomy was performed by Fedor Krause collaborated with neuropathologist Hermann Oppenheim in 1908 at Berlin Augusta Hospital ${ }^{12)}$. In 1934, open discectomy technique procedure to treat ruptured disc without the assistance of the magnifying device was initially advocated by Mixter and Barr, this is a qualitative leap in the history of spine surgery and they described that the sciatica was associated with the compression of nerve root and spinal cord $^{13)}$. In 1939, Love J reported a modified discectomy via interlaminar approach which minimized the bone resection while removing herniation. Although this technique reduces the incidence of postoperative complications associated with total laminectomy, it cannot avoid the issues of postoperative disc degeneration and postoperative back pain ${ }^{14)}$. One year later, Valls et al. reported a percutaneous technique for aspiration biopsy of the vertebral bodies, which laid the foundation for minimally invasive spine surgery ${ }^{15)}$. Later, under the unremitting research on chymopapain chemonucleolysis and the first animal experiment with disc injection by chymopapain was first described by Smith in 1964, and the results shows there is no surrounding tissues dissolution while nucleus pulposus was dissolved byenzy $\mathrm{me}^{16)}$; however, a 12 years clinical experiment reported a series of serious complications in patients who received disc injections with chymopapain, such as sensitivity reactions and neurological reactions ${ }^{17)}$.

In 1975, percutaneous mechanical nucleotomy through foraminal approach under local anesthesia was first introduced by Hijikata and Yamagishi ${ }^{18)}$. Then in 1977, Yasargil reported the first article to perform lumbar discectomy with an operating microscope which is the most popularized surgical-assisted tool until now $^{19)}$. In the subsequent years, CT scanning with myelography significantly improved the evaluation of lumbar degenerative disc disease. In 1983, Weber designed a randomized experiment according to the surgical indications of patients and compared the result between the surgical and conservative treatment with a long-term follow-up, meanwhile disclosed the natural history of the disc herniation ${ }^{20)}$. In 1991, Kambin introduced the foraminal triangular zone as a safe transforaminal approach to access the spinal canal ${ }^{21,22)}$. Assisted with arthroscopy, decompression was successfully performed while avoiding the risk of iatrogenic nerve root injury, and then he analyzed the failure procedures and surgery-related complications in a series of 100 patients. In 1997, Yeung described the integrated, multichannel, wide-angled Yeung Endoscopic spine system which was approved by the US FDA and marketed in the USA. After one year later, Yeung introduced selective endoscopic discectomy to treat herniated discs. However, the hindrance of bilateral iliac crest of L5/S1 restricts the mobility of the endoscopy manipulation via the transforaminal approach. In 2006, the interlaminar approach was early described by Ruetten and Choi to treat lumbar disc herniation and spinal stenosis; The interlaminar approach reduce the bony work on the interlaminar window and protect the natural anatomical integrity of lamina, especially in L5/S1 level ${ }^{22,23)}$. By 2008, the first prospective randomized clinical trial reported by Ruetten to compare the effect of endoscopy with microsurgery on lumbar discectomy. In the past 20 years, full endoscopic spine surgery has been rapidly developed and has been popularized in parts of countries. And the most development of spinal endoscopic surgery is mainly concentrated in East Asia and Germany (Figure 1).

In addition, we also research the top five countries which publish the most articles and hold the most academic activities in the field of spinal endoscopic surgery each year. In the table, we summarize the different timelines of the top 5 major countries in the field of full endoscopic spine surgery. We find that Germany, whether IELD, TELD or full-endoscopic Lumbar Laminotomy (ELL), was the first country to develop these three technologies before the millennium compared to other countries. South Korea is the second only to Germany in the technical publication of IELD and TELD, whereas the first report of ELL is the latest among all regions in South Korea. There was a long interval comparing the first report of the three technologies in Japan and China with Germany, especially in TELD and ELL.

\section{Craftsmanship of IELD Technique}

\section{1) Anesthesia}

Generally, most surgeons will choose general anesthesia as an anesthetic method, and it can reduce the back and leg pain caused by the patient's nerve structure manipulation. However, patients under general anesthesia not only need preoperative fasting and postoperative recovery, but also cannot identify whether there is excessive stretching of nerve root and the sufficiency of nerve decompression intraoperatively. The first prospective controlled study under local and general anesthesia in L5-S1 was reported by HT Chen et al. Authors concluded that there is no clinical parameter difference between two anesthesia methods; however local anesthesia significantly shortens the hospital stays. Although local anesthesia will bring pain to the patient's lower back or legs during the manipulation of the nerve tissue, it allows real-time intraoperative monitoring of clinical conditions to prevent accidental iatrogenic nerve damage ${ }^{25)}$. Feng et al. 


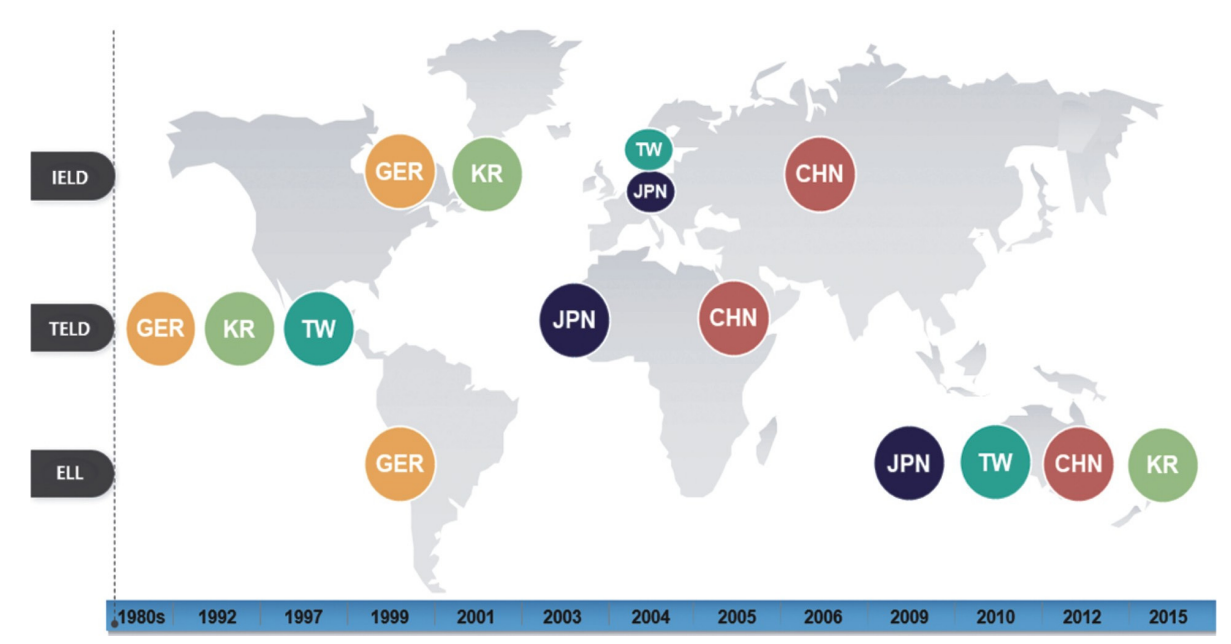

Figure 1. The different timeline shows the historical pioneer of the lumbar discectomy.

introduced a gradient local anesthesia for 50 patients with L5/S1 disc herniation. First, select the puncture point where close to the base of the spinous process at L5/S1 central intervertebral space, and then perform step by step anes- thesia on the skin, subcutaneous tissue, laminae periosteum, ipsilateral facet joint, and outermost layer of ligamentum flavum. Then, complete the anesthesia in the epidural space and perform the endoscopy procedure. The postoperative outcomes showed all of the fifty patients were satisfactory and none of them converted to general anesthesia intraoperatively. Meanwhile, during the operation, patients did not feel pain or only acceptable pain occurred. The author argued that the anesthesia effect of lidocaine alone is insufficient in IELD surgery. Therefore, it is recommended to reduce the amount of saline solution and add ropivacaine, which has a longer half-life period and the function of selective sensory nerve block, to enhance the efficiency of local anesthesia ${ }^{26,27)}$. However, in the similar local anesthesia method reported by Wu et al., traversing nerve root block is added at the proximal side. Although this effectively controls the intrao- perative nerve root pain, it also increases the risk of anesthesia complications caused by overdose of narcotic drugs, such as stethalgia or dyspnea ${ }^{28)}$.

\section{2) Evolution of the Indications}

Traditionally, the lower down bend transverse process, laterally extended isthmus and related large facet joint overlap the disc place from the lateral approach in L5-S1 level. In addition, the height and width dimensions of the intertransverse space is smallest, so it is challenging to remove the fragment through a transforaminal approach by levering without foraminoplasty. Therefore, the interlaminar approach of endoscopy is recommended used in L5/S1 lumbar disc herniation ${ }^{29,30)}$. Tonosu et al. suggested that an intervertebral disc space with a width of at least $20 \mathrm{~mm}$ is necessary for the interlaminar approach; however, when performing an interlaminar approach in a high-level lumbar spine, a cumbersome process of bone resection is required because of the narrow interlaminar space. Meanwhile, the early interlaminar technique is unsuitable for treating patients with spinal instability, seriously scoliosis or high-migrated disc herniation. With the development of endoscopic devices and accumulation of clinician proficiency, the surgical indications for the interlaminar approach gradually continued to expand ${ }^{31)}$.

In general, traditional endoscopic technique is often associated with a high failure rate on high-migrated herniated disc fragments. In order to break the predicament on the treatment of migrated herniation, a series of modified operations have been reported. Lin et al. reported a series of cases with a full-endoscopic interlaminar approach to treat high-grade up-migrated herniation which the extent of migration is beyond the half of infrapedicle level ${ }^{32}$. They directly construct a volcanic-shaped hole on the lamina which is located on the dorsal side of the protrusion to enter the epidural space by means of high-speed burr. Kim et al. illustrated another modified IELD procedure to drill a similar key-hole above the protrusion, and then change to the small-caliber endoscope to pass through the volcanicshaped corridor and remove the high-migrated herniated fragment directly ${ }^{33)}$. The translaminar keyhole approach can not only avoid extensive resection of facet joint and lamina, reduce epidural scar formation, but also preserve the natural anatomical structure between the lamina; it's worth noting that this method is selectively applied to protrusions which located in the shoulder of the nerve root, and not recommended for protrusions that are still connected to the intervertebral disc. Meanwhile, the inner cortical side of the lamina at high-migrated level relatively lacks the protection of the ligamentum flavum which has potential risk of iatrogenic dural damage. Another research reported by Choi retrospectively anatomically analyses the indication of patient selection of IELD, the results statistically indicate that the L5/S1 highly migrated herniation is included in the indications ${ }^{34)}$.

Patients with degenerative lumbar spinal stenosis have hypertrophy of the ligamentum flavum, facet joint hypertrophy, and compression of lumbar disc herniation accompanied by osteophytes, these multiple stenosis factors not only increase the workload of the endoscopy, but also may bring the risk of incomplete decom 
pression in the ipsilateral lateral recess. With the development of the endoscopic instruments, the treatment of lumbar stenosis has been achieved ${ }^{35,36)}$. Chen et al. introduced a hybrid interlaminar technique, a V-shaped bony boundary which consists of the medial facet joint with caudal lamina is drilled by high-speed burr to expose the margin of ligamentum flavum and the area of lateral recess to relieve the pressure of nerve root. After enlargement of the operation window, changing to the small diameter endoscope to perform discectomy and decompress the nerve structure ${ }^{37)}$. A recent meta-analysis was reported by Lee to research the feasibility of IELD on the treatment of spinal stenosis, the Oswestry Disability Index and visual analogue scale scores improved significantly and surpass the criteria for minimal clinically important difference in statistically ${ }^{38}$.

In the early cases, it is a contraindication for IELD to treat fora minal stenosis because of the anatomical considerations of the articular process. Especially in the elderly, there will be pathological changes of facet hypertrophy. Wu illustrated an endoscopic contralateral interlaminar approach technique which passes through the sublaminar corridor for the treatment of patients with foraminal and extraforaminal stenosis ${ }^{39}$. Through this suspension corridor between the nerve element and the dorsal lamina, the whole ligamentum flavum from the cranial, caudal and lateral extension side are removed. Then the nerve root decompression and contralateral discectomy can be performed by foraminotomy $\mathrm{y}^{24)}$. The drilling path from ventral lower half of the lamina to superior articular process not only maintains the stability of the vertebral structure but reduces the intraoperative nerve injury because of the retract of nerve structure. Although this needs an extremely high degree of proficiency for the operation of endoscopy, it also broadens the traditional view of contraindications of interlaminar endoscopy.

\section{3) Tailored Procedures}

Judging the projection of the nerve roots on the dorsal lamina under the assist of imaging will determine the extent of laminotomy and avoid unnecessary bone resection. Koga clarified a minimal laminotomy technique to selective unroofing of the laminar or medial facet, the author concluded that the medial margin of the superior articular process or the caudal margin of the upper laminae should be the main target point of laminectomy for the patient who has related narrow interlaminar width or the concave shape of the upper laminae, it can prevent postoperative segmental instability and cicatrization ${ }^{40}$. In addition, the decision of surgical path should not be based solely on the natural anatomical corridor, but also needs to be considered of the specific location of the protrusion. Choi $\mathrm{G}$ recommends to customize the entry point according to the anatomic structure framed by nerve root, thecal sac and lamina which construct two different safety triangle paths based on the positions of the axilla and shoulder protrusions, and then the endoscope is introduced to remove stained protrusion from the spinal canal with constant inflow of antibiotic saline $\mathrm{e}^{23)}$.

For the removal of central disc herniation in endoscopy, it is an intractable issue from the posterior to reach the target point.
Choi et al. reported that the facetectomy is an effective means to allow the working cannula tilting appropriately and access the central of the spinal canal, thus the instrument compression on the dural sac and cauda equina caused by displacing the working cannula medially is avoided ${ }^{34)}$. On the contrary, in the case of a herniation located at the shoulder area, an inadequate medial facetectomy may have potential to damage the nerve tissue. Kong et al. reported that the different puncture directions can be selected according to the positions of the herniated $\operatorname{disc}^{41)}$; For the intervertebral disc shoulder or axillary disc herniation, the nerve root can be decompressed by separating nerve root medially or direct utilizing the nerve root-dural sac fan-shaped area which passively expanded by the protrusion. The puncture point for shoulder herniation should be close to the medial Crosspoint of the articular process while the approach near to the spinous process is suitable for axillary herniation. If it is difficult to distinguish the starting point of the protrusion because of severe compression, first explore the outward of the dural sac, then follow the outer edge of the dural sac to gradually find the starting point where it intersects with the herniation; When indistinct instrument positioning happens; seeking for the anatomical landmarks, such as the foundation of the spinous process or walking along the surface of the intervertebral disc to find the exit of the protrusion. Therefore, the above skills not only realize the quick puncture from the surface to the target area but also consider the safety of decompression.

\section{4) Revision}

How to accurately treat patients with recurrence of disc herniation and minimize the secondary injury during revision surgery has always been an intractable issue. Conventional disc revision of discectomy surgery often results in incidental durotomy and cerebrospinal fluid leakage interference by epidural scarring, and segmental instability caused by excessive bony work $^{42}$. Meanwhile, it will also significantly increase the rate of postoperative infection ${ }^{43}$. L4-5 and L5-S1 are the most susceptible levels with lumbar disc herniation, and there are $12.11 \%$ of patients with recurrent herniation who have undergone conservative or surgical treatment ${ }^{44}$. The average age of spine patients is widespread older which is one of the predisposing factors for the frustrated operation outcomes ${ }^{45}$. According to early descriptions, radical discectomy is used to curette the cartilage endplate and nucleus pulposus to reduce the recurrence rate of herniation patients; however, traditional secondary revision significantly increased the infection rate of the vertebrae and adjacent tissues. Goker et al. reported a series of 60 patients who accepted IELD revision surgery after Microsurgical discectomy or IELD ${ }^{46}$. During the procedure, the medial area of the articular process was first identified to ensure the positioning of the nerve root, then twist working channel medially to distinguish the disc level with protrusion. There were no surgery-related complications occurred, and the clinical outcomes improved significantly. Wasinpongwanich et al. reported a series of 545 international patient retrospective reviews of IELD postoperative outcomes ${ }^{44}$. The recurrence rate of all patients after being treated with IELD surgery 
Table 1. The table shows the year of the first surgery in top 5 major countries in the field of full-endoscopic spinal surgery

\begin{tabular}{l|c|c|c}
\hline \hline & TELD & IELD & Endoscopic Lumbar Laminotomy \\
\hline China & 2005 & 2006 & 2009 \\
Japan & 2003 & 2004 & 2015 \\
South Korea & 1992 & 2001 & 2010 \\
Taiwan & 1997 & 2004 & 1999 \\
Germany & $1980 s$ & 1999 & \\
\hline
\end{tabular}

TELD, transforaminal full-endoscopic lumbar discectomy; IELD, interlaminar full-endoscopic lumbar discectomy

is only $7.30 \%$ while the average recurrence time is much longer than Yao's TELD clinical results. Author argued that the recurrence rate is not related to ethnicity difference statistically ${ }^{30}$. Although IELD surgery has a relatively low recurrence rate and a long recurrence interval, it also may cause intraoperative nerve damage and postoperative neurological dysfunction like extremity paraesthesia due to the visual confusion under the magnified field of camera in the second revision ${ }^{47)}$. Certainly, this can be avoided as much as possible by carefully stripping tissue which connects to the scar tissue and grinding the adjacent bone window to create a clear visual field. Furthermore, blunt dissect the ligamentum flavum as much as possible instead of resecting the ligamentum flavum to preserve the natural anatomical hierarchy, and it will be helpful for possible future secondary surger ${ }^{23,48)}$. For patients with extremely complex conditions like severe adhesion or vision obscuring because of bleeding from the large epidural vessels, TELD or microscopic discectomy should be considered to avoid the complexity of secondary invasion caused by scar formation ${ }^{23)}$.

In addition, focusing on recurrence risk factors assists spine surgeons to anticipate the potential risk of recurrence, and takes appropriate measures in advance to reduce the incidence rate. Yao et al. reported a research for the recurrence risk factors of IELD and argued that obesity is the significant risk factor of recurrence ${ }^{45)}$. However, Wasinpongwanich's survival analysis showed that patients with high BMl and low BMl have similar recurrence rates. Later, Kim investigated the risk factors of patients with early recurrence in six months postoperatively ${ }^{49)}$. The results show that whether to perform radical annulotomy or surgical approach have no difference for the recurrence while BMl, disc degeneration scale based on Pfirrmann grading system, early ambulation, orthosis application and the number of disc herniation levels impact the incidence of recurrence. Meanwhile, it also found that the recurrence rate of the intervertebral disc collapse height less than $80 \%$ is twice as that of more than $80 \%$, which is in line with Axelsson's viewpoint that the degenerated segment with less disc height change may cause more instability than that of the collapsed disc ${ }^{50}$.

\section{IELD Versus TELD}

Endoscopic lumbar discectomy gradually evolves into a mainst-
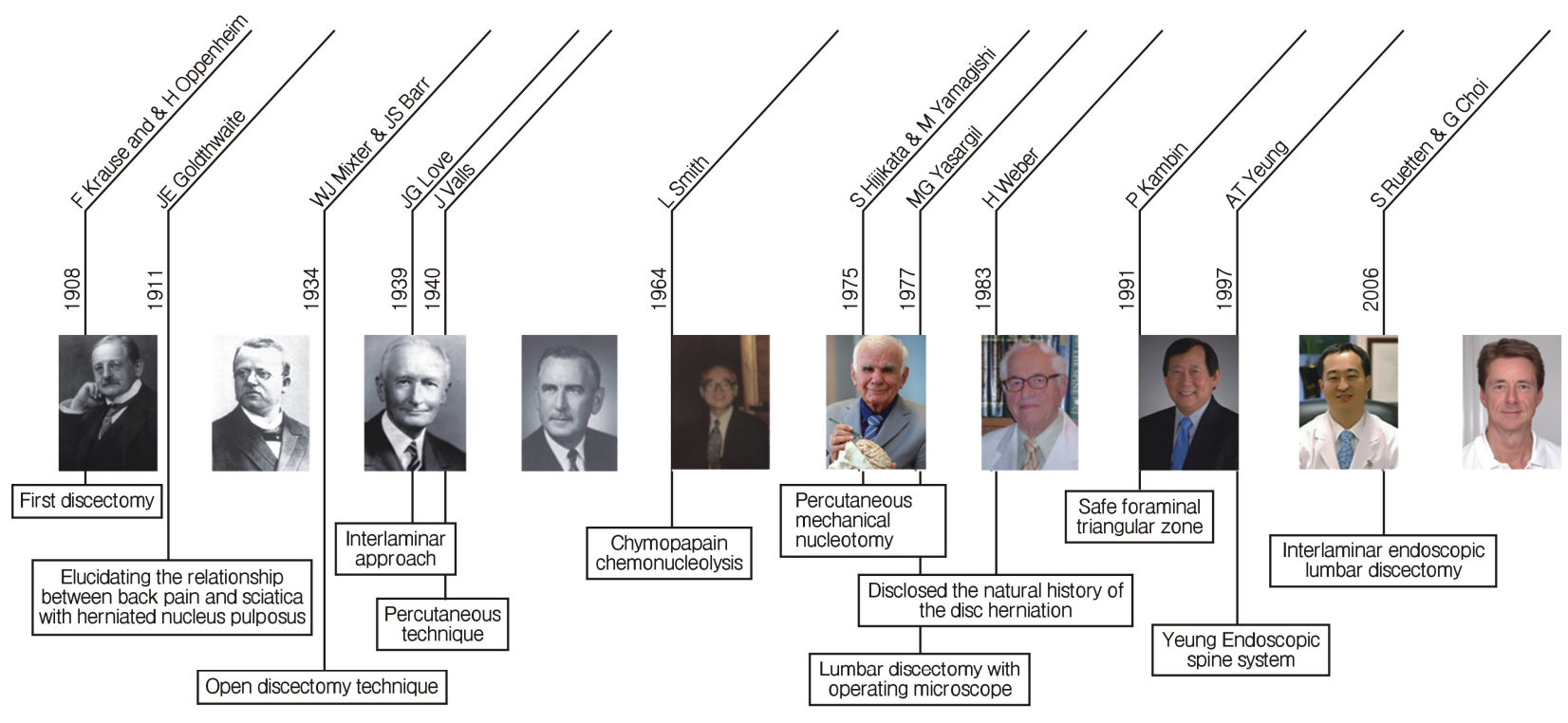

98 yrs

Figure 2. The diagram shows the year of the first surgery in top 5 major countries in the field of full-endoscopic spinal surgery (TELD, transforaminal full-endoscopic lumbar discectomy; IELD, interlaminar full-endoscopic lumbar discectomy; ELL, full-endoscopic Lumbar Laminotomy). 
ream treatment for lumbar disc herniation. Based on the different approach for lumbar discectomy, it can be divided into transforaminal and interlaminar discectomy. IELD is the alternative technique of TELD and is well addressed for lower lumbar segment disc herniation which is located at subarticular or lateral recess areas. In the recent years, plenty of reviews have been reported comparing and trying to clarify the advantages of IELD over TELD from data analysis. Huang et al. reported a meta-analysis to comprehensively analyse the clinical effects of TELD and IELD in the treatment of LDH in 974 patients ${ }^{51)}$. The results showed that although the two approaches have no statistical difference in various clinical outcome scores, recovery time and recurrence rate, TELD has advantages in reducing postoperative complications, and IELD has advantages in reducing intraoperative bleeding, shortening the time of fluoroscopy and operation. A recent meta-analysis study in a series of 3294 patients to compare the efficacy of TELD with IELD, it showed that the visual analog scale scores are lower than IELD, and the author described that the visual analog scale scores reduction is related to the thoroughness of herniation treatment and to the extent of surgical damage ${ }^{52}$. We typically think about IELD shows advantages in avoiding the approach barrier of iliac crest level compared to transforaminal approach; however, choi reported that the decision of surgical approach for $\mathrm{L} 5 / \mathrm{S} 1$ not depends on the height of intervertebral disc and iliac crest, it is statistically related to the location and the type of the disc herniation as well as the high-grade migration ${ }^{34)}$. In terms of radiation exposure, TELD consumes more time than IELD on puncture to ensure the nerve roots are not disturbed, therefore, it will increase exposure to radiation ${ }^{53)}$. One prospective randomized controlled study reported by $\mathrm{Nie}$, he illustrated that even the transforaminal and interlaminar approach have similar clinical parameters, but the fluoroscopy time in the interlaminar approach is significantly less than transforaminal approach ${ }^{54)}$. In addition, surgical decision-making requires surgeons to evaluate the most effective intervention for the patient, and to compare the extent of the value that can be produced in terms of cost and health impact between multiple interventions. Wang et al. adopted macro-costing way to conduct a cost-utility analysis between TELD or IELD, and found that although the total cost of patients and postoperative quality of life were not statistically different, the cost of IELD surgical equipment, surgical material, anesthesia are higher than that of TELD ${ }^{55}$.

\section{Complication}

Surgeons should be alert on the potential occurrence of complications in the IELD, albeit a multiple advantage in the treatment of lumbar disc herniation. In particular, the pressure of the irrigation fluid system may increase epidural pressure and obscure concealed hemorrhage, which may cause postoperative epidural hematoma. Excessive removal of the anterior annulus structure removal may result in penetration of the anterior longitudinal ligament and damage to internal organs or blood vessels, additionally, although the endoscopic radiofrequency has been manipulated under cold saline irrigation, impro- per use can still cause the risk of thermal damage to nerve tissue ${ }^{23}$. One of the meta-analyses which compared TELD versus IELD for lumbar disc herniation reported that although two groups reported no significant rate difference in recurrence and revision, the incidence of complications in IELD group more than TELD group statistically $y^{56}$. Author speculated that the potential reason for the relatively high rate of interlaminar approach postoperative complications might be the excessive retraction of the neural structure. However, these complications in endoscopy can be avoided with the accumulation of experience and continuous improve surgical skill for individual surgeons ${ }^{57)}$; The patch-blocking dura repair technique used in open surgery can be transferred by the application of collagen-fibrin sealant patch in endoscopy for small incidental durotomy; Setting proper water pressure of irrigation system can ensure the normal cerebrospinal fluid flows while discover the bleeding points in time; Before removing of the working cannula, the pulsation of the dural sac should be detected and check the freedom of the nerve root carefully, then performing sufficient hemostasis to prevent postoperative hematoma.

\section{Learning Curve}

For less experienced residents, it is a challenge for spinal manipulation in distinguishing the anatomical structure and identifying the obscure positioning of the instrument simultaneously in the full-endoscopy operation ${ }^{58)}$. At the same time, it will bring a higher probability of complications ${ }^{59}$. Son statistically analyzed the operation time to assess the learning curve between IELD and open lumbar microdiscectomy, the author argued that IELD group have significantly shorter operation time; however, the turning point of the learn curve of operation time is the $18^{\text {th }}$ case in IELD and the $10^{\text {th }}$ case in open microdiscectomy due to the cumulative proficiency ${ }^{60)}$. Although the learning curve of endoscopic spine surgery is steep from traditional and microscopic surgery, the anatomical structure of the approach from the interlaminar space is more familiar to orthopedic and neurosurgery residencies than that of the foraminal approach. Meanwhile, carefully analyzing the imaging before surgery, combining with a navigation system and skillfully manipulating instruments can ensure safer and thorough decompression. Indubitably, a favorable clinical result requires an experienced team to perform.

\section{CONCLUSION}

In the past decades, with the accumulation of spine surgeons' understanding and experience of endoscopy, the indications for IELD is broaden in the treatment of patients with symptomatic disc herniation. The results of articles reported have also confirmed that endoscopic technology is a safe and effective technique of spine surgery for patients who meet its indications. The endoscopy technology directly uses the natural anatomy of the interlaminar window to avoid the extra damage caused by surgical invasion to the greatest extent. The learning curve of endoscopy has shallow learning curve with the development of imaging 
systems in assistive technology. Furthermore, surgeons should be alert to a series of complications, and perform preventive measures to avoid unnecessary iatrogenic injuries.

\section{CONFLICT OF INTEREST}

The corresponding author, Jin-Sung Kim, is a consultant of Richard Wolf, GmbH, Germany and Elliquence, LLC, USA

\section{REFERENCES}

1. Hoy D, Brooks P, Blyth F, Buchbinder R: The Epidemiology of low back pain. Best Practice \& Research: Clinical Rheumatology 24(6):769-781, 2010

2. Amin RM, Andrade NS, Neuman BJ: Lumbar Disc Herniation. Curr Rev Musculoskelet Med 10(4):507-516, 2017

3. Andersson GB: Epidemiological features of chronic low-back pain. Lancet 354(9178):581-585, 1999

4. Konstantinou K, Dunn KM: Sciatica: Review of epidemiological studies and prevalence estimates. Spine (Phila Pa 1976) 33(22):24642472, 2008

5. Fardon DF, Williams AL, Dohring EJ, Murtagh FR, Gabriel Rothman SL, Sze GK: Lumbar disc nomenclature: Version 2.0: Recommendations of the combined task forces of the North AmericanSpine Society, the American Society of Spine Radiology and the American Society of Neuroradiology. The Spine Journal 14(11): 2525-2545, 2014

6. Giurazza F, Guarnieri G, Murphy KJ, Muto M: Intradiscal O2-O3: Rationale, Injection Technique, Short- and Long-term Outcomes for the Treatment of Low Back Pain Due to Disc Herniation. Cancer Association of Radiologists Journal 68(2):171-177, 2017

7. Perri M, Marsecano C, V arrassi M, Giordano AV, Splendiani A, et al.: Indications and efficacy of $\mathrm{O} 2-\mathrm{O} 3$ intradiscal versus steroid intraforaminal injection in different types of disco vertebral pathologies: A prospective randomized double-blind trial with 517 patients. La Radiologia Medica 121(6):463-471, 2016

8. Gibson JN, Waddell G: Surgical interventions for lumbar disc prolapse: Updated Cochrane Review. Spine (Phila Pa 1976) 32(16): 1735-1747, 2007

9. Herkowitz HN, Garfin SR, Eismont FJ, Bell GR, Balderston RA: Lumbar disk herniations. In: Rothman-Simeone The Spine. $6^{\text {th }}$ edn. Saunders, 2011

10. Ahn Y, Lee SH, Lee JH, et al.: Transforaminal percutaneous endoscopic lumbar discectomy for upper lumbar disc herniation: Clinical outcome, prognostic factors, and technical consideration. Acta Neurochir 151(3):199-206, 2009

11. Chen KT, Jabri H, Lokanath YK, Song MS, Kim JS: The evolution of interlaminar endoscopic spine surgery. J Spine Surg 6(2): 502-512, 2020

12. Bacigaluppi S, Bragazzi NL, Martini M: Fedor Krause (18571937): the father of neurosurgery. Neurosurg Rev 43(6):14431449, 2020

13. Mixter WJ, Barr JS: Rupture of the intervertebral disc with involvement of the spinal canal. The New England Journal of Medicine 211:210-215, 1934

14. Love JG: Removal of protruded intervertebral discs without laminectomy. Proc Staff Meeting. Mayo Clinic 14:800, 1939
15. Valls J, Ottolenghi CE, Schajowicz F: Aspiration biopsy in diagnosis of lesions of vertebral bodies. J Am Med Assoc136(6):376382, 1948

16. Smith L: Enzyme Dissolution of the Nucleus Pulposus in Humans. JAMA Jan 11(187):137-140, 1964

17. Watts C: Complications of chemonucleolysis for lumbar disc disease. Neurosurgery 1(1):2-5, 1977

18. Hijikata S, Yamagishi M, Nakayama T, Oomori K: Percutaneous diskectomy: A new treatment method for lumbar disc herniation. J Toden Hosp 5:5-13, 1975

19. Yasargil MG, Vise WM, Bader DC: Technical adjuncts in neurosurgery. Surg Neurol 8(5):331-336, 1977

20. Weber H: Lumbar disc herniation. A controlled, prospective study with ten years of observation. Spine (Phila Pa 1976) 8(2):131140, 1983

21. Kambin P: Arthroscopic microdiscectomy. The Spine Journal 3 (3):60-64, 2003

22. Ruetten S, Komp M, Godolias G: A New full-endoscopic technique for the interlaminar operation of lumbar disc herniations using 6-mm endoscopes: Prospective 2-year results of 331 patients. Minim Invasive Neurosurg 49(2):80-87, 2006

23. Choi G, Lee SH, Raiturker PP, Lee S, Chae YS: Percutaneous endoscopic interlaminar discectomy for intracanalicular disc herniations at L5-S1 using a rigid working channel endoscope. Neurosurgery 58(1 Suppl): ONS59-68, 2006

24. Ebraheim NA, XU R, Huntoon M, Yeasting RA: Location of the extraforaminal lumbar nerve roots. An anatomic study. Clin Orthop Relat Res 340:230-235, 1997

25. Chen HT, Tsai CH, Chao SC, Kao TH, Chen YJ, Hsu HC, et al: Endoscopic discectomy of L5-S1 disc herniation via an interlaminar approach: Prospective controlled study under local and general anesthesia. Surg Neurol Int 2:93, 2011

26. Feng WL, Yang JS, Wei D, Gong HL, Xi Y, Lv HQ, Wang XG, Xia B, Wei JM: Gradient local anesthesia for percutaneous endoscopic interlaminar discectomy at the L5/S1 level: A feasibility study. J Orthop Surg Res 15(1):413, 2020

27. Hansen TG: Ropivacaine: A pharmacological review. Expert Rev Neurother 4(5):781-791, 2004

28. Wu K, Zhao Y, Feng Z, Hu X, Chen Z, Wang Y: Stepwise Local Anesthesia for Percutaneous Endoscopic Interlaminar Discectomy: Technique Strategy and Clinical Outcomes. World Neurosurg 134:e346-352, 2020

29. Ebraheim NA, XU R, Huntoon M, Yeasting RA: Location of the extraforaminal lumbar nerve roots. An anatomic study. Clin Orthop Relat Res 340:230-235, 1997

30. Reulen HD, Muller AD, Ebeling UD: Misrosurgical anatomy of the lateral approach to extraforaminal lumbar disc herniations. Neurosurgery 39(2):345-351, 1996

31. Tonosu J, Oshima Y, Shiboi R, Hayashi A, Takano Y, Inanami $\mathrm{H}$, et al.: Consideration of proper operative route for interlaminar approach for percutaneous endoscopic lumbar discectomy. J Spine Surg 2(4):281-288, 2016

32. Lin GX, Park CW, Suen TK, Kotheeranurak V, Jun SG, Kim JS: Full Endoscopic Technique for High-Grade Up-Migrated Lumbar Disk Herniation via a Translaminar Keyhole Approach: Preliminary Series and Technical Note. J Neurol Surg A Cent Eur Neurosurg 81(5):379-386, 2020

33. Khandge AV, Kim JS: Modified Interlaminar Endoscopic Lum- 
bar Discectomy for Highly Upmigrated Disc Herniation: A Proctorship Description of the Technique via Translaminar Route. Neurospine 17(Suppl 1): S66-S73, 2020

34. Choi KC, Kim JS, Ryu KS, Kang BU, Ahn Y, Lee SH: Percutaneous endoscopic lumbar discectomy for L5-S1 disc herniation: Transforaminal versus interlaminar approach. Pain Physician 16 (6):547-556, 2013

35. Ohba T, Ebata S, Fujita K, et al.: Characterization of symptomatic lumbar foraminal stenosis by conventional imaging. Eur Spine J 24(10):2269-2275, 2015

36. Hughes A, Makirov SK, Osadchiy V: Measuring spinal canal size in lumbar spinal stenosis: Description of method and preliminary results. Int J Spine Surg 24(9):3, 2015

37. Chen KT, Choi KC, Song MS, Jabri H, Lokanath YK, Kim JS: Hybrid Interlaminar Endoscopic Lumbar Decompression in Disc Herniation Combined with Spinal Stenosis. Oper Neurosurg 8: opaa360, 2020

38. Lee CH, Choi M, Ryu DS, et al.: Efficacy and Safety of Fullendoscopic Decompression via Interlaminar Approach for Central or Lateral Recess Spinal Stenosis of the Lumbar Spine: A Metaanalysis. Spine (Phila Pa 1976) 43(24):1756-1764, 2018

39. Wu PH, Kim HS, Jang IT: How I do it? Uniportal full endoscopic contralateral approach for lumbar foraminal stenosis with double crush syndrome. Acta Neurochir 162(2):305-310, 2020

40. Koga $\mathrm{H}$, Inanami $\mathrm{H}$ : Minimal laminectomy using the interlaminar approach for percutaneous endoscopic lumbar discectomy. Miniinvasive Surg 1:56-62, 2017

41. Kong W, Chen T, Ye S, Wu F, Song Y: Treatment of L5-S1 intervertebral disc herniation with posterior percutaneous full-endoscopic discectomy by grafting tubes at various positions via an interlaminar approach. BMC Surg 19(1):124, 2019

42. Ahn Y, Lee SH, Park WM, Lee HY, Shin SW, Kang HY: Percutaneous endoscopic lumbar discectomy for recurrent disc herniation: Surgical technique, outcome, and prognostic factors of 43 consecutive cases. Spine (Phila Pa 1976) 29(16):E326-332, 2004

43. Yorukoglu AG, Goker B, T ahta A, Akcakaya MO, et al.: Fully endoscopic interlaminar and transforaminal lumbar discectomy: Analysis of 47 complications encountered in a series of 835 patients. Neurocirugia 28(5):235-241, 2017

44. Wasinpongwanich K, Pongpirul K, Lwin KMM, Kesornsak W, Kuansongtham V, Ruetten S: Full-Endoscopic Interlaminar Lumbar Discectomy: Retrospective Review of Clinical Results and Complications in 545 International Patients. World Neurosurg 132:e922-e928, 2019

45. Yao Y, Liu H, Zhang H, et al.: Risk Factors for Recurrent Herniation After Percutaneous Endoscopic Lumbar Discectomy. World Neurosurg 100:1-6, 2017

46. Goker B, Aydin S: Endoscopic Surgery for Recurrent Disc Herniation After Microscopic or Endoscopic Lumbar Discectomy. Turk Neurosurg 30(1):112-118, 2020

47. Ruetten S, Komp M, Merk H, Godolias G: Recurrent lumbar disc herniation after conventional discectomy: A prospective, randomized study comparing full-endoscopic interlaminar and transforaminal versus microsurgical revision. J Spinal Disord Tech 22(2):122-129, 2009

48. Boeree N: The Reduction of Peridural Fibrosis, in Gunzburg R (ed): Lumbar Disc Herniation. Philadelphia, Lippincott Williams \& Wilkins 2002:185-196, 2002

49. Kim HS, You JD, Ju CI: Predictive Scoring and Risk Factors of Early Recurrence after Percutaneous Endoscopic Lumbar Discectomy. Biomed Res Int 2019:6492675, 2019

50. P Axelsson, BS Karlsson: Intervertebral mobility in the progressive degenerative process. A radiostereometric analysis. European Spine Journal 13(6):567-572, 2004

51. Huang Y, Yin J, Sun Z, Song S, Zhuang Y, Liu X, Du S, Rui Y: Percutaneous endoscopic lumbar discectomy for $\mathrm{LDH}$ via a transforaminal approach versus an interlaminar approach: A metaanalysis. Orthopade 49(4):338-349, 2020

52. Chen P, Hu Y, Li Z: Percutaneous endoscopic transforaminal discectomy precedes interlaminar discectomy in the efficacy and safety for lumbar disc herniation. Biosci Rep 15;39(2): BSR 20181866, 2019

53. Wu R, Liao X, Xia H: Radiation exposure to the surgeon during ultrasound-assisted transforaminal percutaneous endoscopic lumbar discectomy: A prospective study. World Neurosurg 101:658665, 2017

54. Nie H, Zeng J, Song Y, Chen G, Wang X, Li Z, et al.: Percutaneous Endoscopic Lumbar Discectomy for L5-S1 Disc Herniation Via an Interlaminar Approach Versus a Transforaminal Approach: A Prospective Randomized Controlled Study With 2Year Follow Up. Spine (Phila Pa 1976) 41:B30-B37, 2016

55. Wang D, Xie W, Cao W, He S, Fan G, Zhang H: A Cost-utility Analysis of Percutaneous Endoscopic Lumbar Discectomy for L5S1 Lumbar Disc Herniation: Transforaminal Versus Interlaminar. Spine (Phila Pa 1976) 44(8):563-570, 2019

56. McAfee PC, Phillips FM, Andersson G, Buvenenadran A, Kim CW, Lauryssen C, et al.: Minimally invasive spine surgery. Spine (Phila Pa 1976) 15(35):S271-S273, 2010

57. Wu PH, Kim HS, Jang IT: A Narrative Review of Development of Full-Endoscopic Lumbar Spine Surgery. Neurospine 17 (Suppl 1):S20-S33, 2020

58. Wang K, Hong X, Zhou BY, Bao JP, Xie XH, Wang F, et al.: Evaluation of transforaminal endoscopic lumbar discectomy in the treatment of lumbar disc herniation. Int Orthop 39(8):15991604, 2015

59. Yorukoglu AG, Goker B, Tahta A, Akcakaya MO, et al.: Fully endoscopic interlaminar and transforaminal lumbar discectomy: Analysis of 47 complications encountered in a series of 835 patients. Neurocirugia 28(5):235-241, 2017

60. Son S, Ahn Y, Lee SG, Kim WK: Learning curve of percutaneous endoscopic interlaminar lumbar discectomy versus open lumbar microdiscectomy at the L5-S1 level. PLOS One 30;15(7):e0236296, 2020 